\title{
Inhibitors of PD-1/PD-L1 and ERK1/2 impede the proliferation of receptor positive and triple-negative breast cancer cell lines
}

\author{
Karen Bräutigam ${ }^{1}$ (D) Elodie Kabore-Wolff ${ }^{1}$ Ahmad Fawzi Hussain ${ }^{2} \cdot$ Stephan Polack $^{1} \cdot$ Achim Rody $^{1} \cdot$ Lars Hanker $^{1}$. \\ Frank Köster ${ }^{1}$
}

Received: 27 April 2021 / Accepted: 10 June 2021 / Published online: 29 June 2021

(c) The Author(s) 2021

\begin{abstract}
Purpose Triple-negative breast cancer (TNBC) is characterized by an unfavorable prognosis and missing systemic therapeutic approaches beside chemotherapy. Targeting the immune checkpoint PD-1/PD-L1 showed promising results in breast cancer and especially in TNBC. The extracellular signal-regulated kinase 1/2 (ERK1/2) is an important driver of carcinogenesis. Here, the effect of combined PD-1/PD-L1 and ERK1/2 inhibitor treatment is investigated of cell growth and intracellular impact of breast cancer cell lines.

Methods $\mathrm{The}_{\mathrm{IC}}$ values of each inhibitor and the effect of combined treatment were determined in three TNBC cell lines of different subtypes and one non-TNBC cell line. Phospho-specific antibodies were used in western blot analyses to investigate an effect on ERK1/2 activation. Expressions of immune modulatory and cell cycle-associated genes were examined by quantitative reverse transcription PCR.

Results Both inhibitors PD-1/PD-L1 and ERK1/2 impeded the proliferation of TNBC to a higher extent than of non-TNBC. By combined treatment, cell lines were inhibited either synergistically or additively. ERK1/2 and S6 phosphorylation were reduced and expressions of c-Fos and FosL were diminished after ERK1/2 inhibitor as single and combined treatment. Between genes involved in immune modulation, IL-8 was upregulated in TNBC cells after combined treatment.

Conclusion In conclusion, combination of PD-1/PD-L1 and ERK1/2 inhibitors showed favorable effects for a new therapy strategy, with better results in TNBC cell lines than in non-TNBC cells. The effects have to be validated in models that can reflect the interaction between immune and tumor cells like the situation in the tumor micro-environment.
\end{abstract}

Keywords Triple-negative breast cancer $\cdot$ Immune checkpoint inhibitor $\cdot$ ERK inhibitor $\cdot$ Proliferation $\cdot$ Combined therapy

\section{Introduction}

Breast cancer is the most common cancer among women in Germany and worldwide (Siegel et al. 2019). 80-85\% of breast cancers display hormone receptor expression and/or overexpression of Her-2 receptor (Cancer Genome Atlas 2012). Receptor-positive breast cancer subtypes are

Lars Hanker and Frank Köster contributed equally to the study.

Karen Bräutigam

karen.braeutigam@uksh.de

1 Department of Gynecology and Obstetrics, University Medical Center Schleswig-Holstein, Campus Lübeck, Lübeck, Germany

2 Department of Gynecology and Obstetrics, Medical Faculty, Justus-Liebig-University Giessen, Giessen, Germany predictive for endocrine therapy and Her- 2 receptor-targeted antibody therapy. $15-20 \%$ of breast cancer cells express none of these receptors and are called triple-negative breast cancer (TNBC). TNBC is associated with higher recurrence and metastasis rates and lower overall survival. Furthermore, the treatment options besides chemotherapy are limited (Dent et al. 2007). TNBCs are a heterogeneous group subdivided into 4 subtypes: basal-like1 (BL1), basal-like2 (BL2), mesenchymal (M) and luminal androgen receptor (LAR) subtype (Lehmann et al. 2016).

The mitogen-activated protein kinases (MAPK) pathways are intracellular signal cascades that regulate multiple cell processes by consecutive phosphorylation of signal transduction proteins. The RAS/RAF/MEK/ERK pathway is the classical MAPK pathway activated by growth factors, cytokines, integrins as well as steroids. The MAPK pathway regulates cell proliferation, migration and angiogenesis 
(Santen et al. 2002). Several studies used small molecule drugs to inhibit the MAPK pathway in various tumor entities, but results for specific ERK1/2 inhibitors in breast cancer are rare.

The selective ERK inhibitor SCH772984, used in this study, not only impedes dose-dependently the phosphorylation of ERK, but also the phosphorylation of S6, one of its downstream targets (Meyuhas 2015).

Besides the MAPK pathway, the STAT3 (signal transducer and activator of transcription 3)-pathway plays an important role in progression and metastasis of breast cancer by promoting angiogenesis and is increasingly phosphorylated in ca. $40 \%$ of breast cancers (Chang et al. 2013).

PD-1 (programmed cell death protein 1) is a transmembrane receptor expressed on immune cells representing an immune checkpoint (Arasanz et al. 2017). PD-1 interacts with two ligands, PD-L1 and 2 (programmed cell death 1 ligand 1 and 2). PD-L1 expression is increased on breast cancer cells and is correlated with tumors having a low prognosis. PD-L1 is more abundant in the subgroup of TNBC (Gatalica et al. 2014; Migali et al. 2016) and expressed in 20-30\% of TNBC (Nanda et al. 2016). PD-L1 modulates the immune response (Patel and Kurzrock 2015). The interaction of PD-L1 with PD-1 inhibits the activation of T lymphocytes and the proliferation of regulatory $\mathrm{T}$ cells infiltrating the tumor and thereby prevents the immune response (tumor immune escape) (Francisco et al. 2010). Many clinical studies described overexpression of PD-L1 in different tumor entities and the anti-tumoral effect by the inhibition of PD-1/ PD-L1 interaction. By now, several immune checkpoint inhibitors are applied in the guidelines of therapy regimes for different cancer types, e.g. melanoma and small cell lung cancer. For TNBC, a study of 902 patients showed an overall advantage for patients treated with a combination of the antiPD-L1 antibody atezolizumab and nab-paclitaxel compared to a therapy with only nab-paclitaxel (Schmid et al. 2020). Atezolizumab is approved for therapy of non-resectable, advanced or metastasized TNBC with PD-L1 positivity in $\geq 1 \%$ of tumor surrounding immune cells (Schmid et al. 2020). Solinas and colleagues described positive effects of combination of PD-1/PD-L1 inhibitors in combination with established therapeutics in breast cancer and especially in TNBC (Solinas et al. 2017). Dependent on the type of cancer, the PD-L1 expression is differently regulated, e. g. in lymphoma and lung cancer PD-L1 expression is elevated by the ALK (anaplastic lymphoma kinase) through STAT3 activation (Marzec et al. 2008). Cui and coworkers described that PD-L1 expression influences EMT (epithelial mesenchymal transition) in oral squamous cell carcinoma. In oral squamous cell carcinoma cell lines, PD-L1 is regulated by ERK and STAT3 signaling. Downregulation of PD-L1 with siRNA was accompanied by downregulation of mesenchymal proteins and decreased phosphorylation of ERK and STAT3 (Cui et al. 2021). Also, ERK mediates PD-L1 expression in NSCLC (non small cell lung carcinoma) cells with ROS 1 fusion: Liu and coworker showed that both ERK phosphorylation and PD-L1 expression were down-regulated after treating HCC78 cells with PD0325901 or U0126 (Liu et al. 2020).

The aim of this study was to investigate the potential impact of either a PD-1/PD-L1 inhibitor or an ERK1/2 inhibitor and the combination of these in TNBC and nonTNBC cell lines.

\section{Materials and methods}

\section{Breast cancer cell lines}

Four established breast cancer cell lines comprising three TNBC: MDA-MB-231 (M); HCC1937 (BL1); HCC1806 (BL2) and one non-TNBC (MCF7) were actually purchased from the American Type Cell Collection (LGC, Wesel, Germany). Cells were cultivated in Dulbecco's modified Eagle's medium (DMEM)) containing 10\% fetal bovine serum, $1 \%$ penicillin-streptomycin (100x) (all from Life Technologies, Darmstadt, Germany) and incubated at $37^{\circ} \mathrm{C}$ in a humidified atmosphere with $5 \% \mathrm{CO}_{2}$.

\section{Compounds}

PD-1/PD-L1 inhibitor 1 (BMS-1) and ERK1/2 inhibitor (SCH772984) were purchased from Selleckchem (Absource Diagnostics GmbH, Munich, Germany) and diluted in dimethyl sulfoxide (DMSO) at $10 \mathrm{mM}$ and $8.51 \mathrm{mM}$, respectively.

\section{Cell proliferation assay}

Cell proliferation was determined by the MTS assay (CellTiter $96^{\circledR}$ AQueous One Solution Reagent, Promega, Walldorf, Germany). A total of $1-2 \times 10^{3}$ cells were plated in $100 \mu \mathrm{l}$ medium in each well of a 96-well plate. After 24 h, PD-1/ PD-L1 and ERK1/2 inhibitor were diluted to the desired concentrations in test medium (DMEM containing 1\% fetal bovine serum) and added as single or as combined treatment in triplicate for $72 \mathrm{~h}$. The MTS assay was repeated at least three times for each cell line to determine the half maximum inhibitory concentration $\left(\mathrm{IC}_{50}\right)$ and an interaction index.

\section{Western blot}

$4 \times 10^{5}$ cells were seeded onto a 6 -well plate for $24 \mathrm{~h}$ and treated for further $24 \mathrm{~h}$. For protein isolation $100 \mu \mathrm{l}$ RIPA buffer (phosphate-buffered saline, $1 \%$ Igepal, $0.5 \%$ sodium deoxycholate, $0.1 \%$ SDS) with freshly added $1 \%$ protease inhibitor and phosphatase inhibitor 2; (Sigma-Aldrich, 
Hamburg, Germany) was used. $20 \mu \mathrm{g}$ per sample were transferred onto polyvinylidene difluoride membranes (Carl Roth, Karlsruhe, Germany). The immune analyses were performed with antibodies in the following mixing ratio: Phospho-p44/42 MAPK (ERK1/2) (1:2,000); p44/42 MAPK (ERK1/2) (1:1,000); Phospho-S6 Ribosomal Protein $(1: 2,000)$; S6 Ribosomal Protein $(1: 1,000)$; PhosphoSTAT3 $(1: 2,000)$; STAT3 $(1: 1,000)$ (all from Cell Signaling Technology, Frankfurt am Main, Germany). For immune detection, we used the Clarity Max Western ECL Substrate (Biorad, Feldkirchen, Germany) and the immune reaction was visualized by the Chemidoc Imaging System and analyzed by Image Lab (Biorad).

\section{RNA isolation, reverse transcription and quantitative real-time polymerase chain reaction (qPCR)}

$4 \times 10^{5}$ cells were grown and treated on a 6-well plate for $24 \mathrm{~h}$ and RNA was extracted using the RNeasy Mini Kit (Qiagen, Hilden, Germany). $1 \mu \mathrm{g}$ RNA was reverse transcribed into cDNA using Superscript-II-Reverse-Transcription from Invitrogen (Fisher Scientific, Schwerte, Germany). QPCR was performed using GoTaq qPCR Master Mix (Promega) and the following primer sequences for analyzing expression of immune modulatory and growth-associated genes: PD-L1: forward 5'-GGACAAGCAGTGACCATCAAG-3', reverse 5'-CCCAGAATTACCAAGTGAGTCCT-3'; interleukin 8 (IL-8): forward 5'-ACTGAGAGTGATTGAGAGTGGAC-3', reverse 5'-AACCCTCTGCACCCAGTTTTC-3'; chemokine (C-X-C Motif) receptor 2 (CXCR2): forward 5'-CCTGTC TTACTTTTCCGAAGGAC-3', reverse 5'-TTGCTGTAT TGTTGCCCATGT-3'; c-Fos: forward 5'-GAGATTGCC AACCTGCTGAA-3', reverse 5'-AGACGAAGGAAGACG TGTAA-3', Fos-like 1 (FosL1): forward 5'-CAGGCGGAG ACTGACAAACTG-3', reverse 5'-TCCTTCCGGGATTTT GCAGAT-3'. PCR product specificity was verified by comparative melting curve analysis. Cycle threshold values of genes of interest were quantified, and normalized to expression of succinate dehydrogenase complex flavoprotein subunit A (SDHA) (forward: 5'-TGGGAACAAGAGGGCATC TG-3', reverse: 5'-CCACCACTGCATCAAATTCATG-3'), and relative expression of genes in drug-treated cells was compared to relative expression of genes in untreated cells using the $2^{-\Delta \Delta C t}$ method (Pfaffl 2001).

\section{Statistical analyses}

All results were expressed as the mean and standard deviation (SD) from three independent experiments. The significance of the differences in cell proliferation using MTS assays after treatment between cell lines was examined in a Student's two-tailed $t$ test. The same test was performed to determine the significance of the differences of single and combined treatment with two inhibitors in MTS assays, western blots and qPCR. Statistical significance: ${ }^{*} p<0.05$, $* * p<0.01, * * * p<0.001$.

\section{Results}

\section{Influence of PD-1/PD-L1 inhibitor and ERK1/2 inhibitor on cell viability of TNBC and the non-TNBC cell lines}

The effect of single treatment of the PD-1/PD-L1 inhibitor and the ERK1/2 inhibitor on cell viability was investigated in all cell lines after $72 \mathrm{~h}$ of treatment and the $\mathrm{IC}_{50}$ values were determined (Table 1). At a concentration of $2.5 \mu \mathrm{M}$, the PD-1/PD-L1 inhibitor significantly impeded the cell viability of TNBC cell lines MDA-MB-231, HCC1937 and HCC1806 to a greater extent compared to the non-TNBC cell line MCF7 (Fig. 1) The sensitivity towards the ERK1/2 inhibitor was significantly higher in the three TNBC cell lines compared with the non-TNBC cell line MCF7, which is shown by $\mathrm{IC}_{50}$ values in Table 1 and Fig. 2. Subsequently, the effect of combined treatment with adapted concentrations of the PD-1/PD-L1 inhibitor and the ERK1/2 inhibitor on cell viability was investigated to calculate the interaction index for both substances. The combination inhibited the proliferation in all cell lines stronger than single treatment (Fig. 3). The concurrent treatment led to an additive effect in the MDA-MB-231 cells $(y=1.02)$. In the basal-like cell lines HCC1937 $(\mathrm{y}=0.88)$ and HCC1806 $(\mathrm{y}=0.85)$ and the MCF7 ( $y=0.76)$ cells, the cell viability was impeded synergistically (Table 2).

Table $1 \mathrm{IC}_{50}$ values estimated by MTS assays

\begin{tabular}{|l|c|c|}
\hline & $\begin{array}{c}\text { PD-1/PD-L1 inhibitor } \\
\mu \mathrm{M}(+/- \text { SD) }\end{array}$ & $\begin{array}{c}\text { ERK1/2 inhibitor } \\
\mu \mathrm{M}(+/-\mathrm{SD})\end{array}$ \\
\hline MCF7 & $3.12(0.44)$ & $27.00(3.55)$ \\
\hline MDA-MB-231 & $1.99(0.39)$ & $6.86(1.50)$ \\
\hline HCC1937 & $1.41(0.33)$ & $7.34(4.11)$ \\
\hline HCC1806 & $1.65(0.48)$ & $5.06(2.58)$ \\
\hline
\end{tabular}

$\mathrm{IC}_{50}$ values $(\mu \mathrm{M})$ of the PD-1/PD-L1 inhibitor 1 and the ERK1/2 inhibitor for the TNBC cells MDA-MB-231, HCC1937, HCC1806 (white), and the non-TNBC cell line MCF7 (gray) were estimated and represented as the mean of three independently performed experiments

$S D$ standard deviation 
Fig. 1 MTS assay after PD-1/ PD-L1 inhibitor 1 treatment. The TNBC cells MDAMB-231, HCC1937, HCC1806, and the non-TNBC cell line MCF7 were treated with rising concentrations of PD-1/ PD-L1 inhibitor 1 for $72 \mathrm{~h}$. Statistical significance of differences between MCF7 and a MDA-MB-231, b HCC1937, and $\mathbf{c} \mathrm{HCC} 1806: * p<0.05$, ** $p<0.01, * * * p<0.001$ ( $t$ test $)$

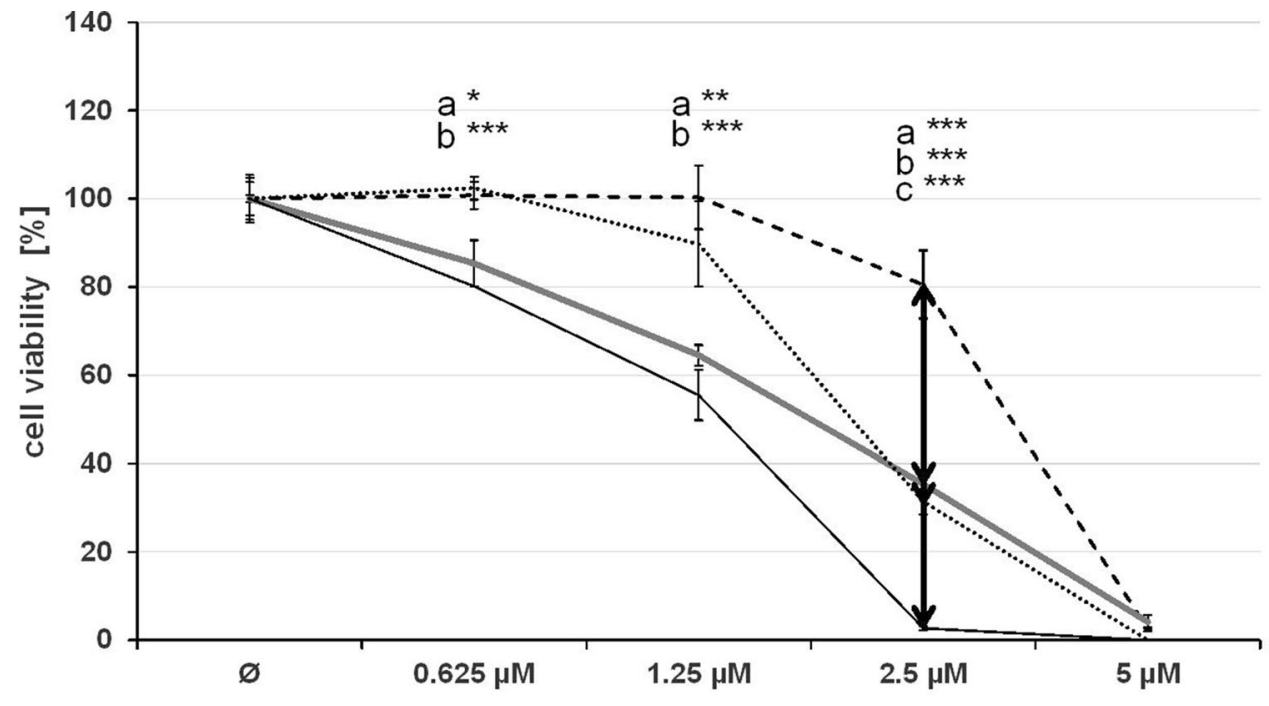

PD-1/PD-L1 inhibitor
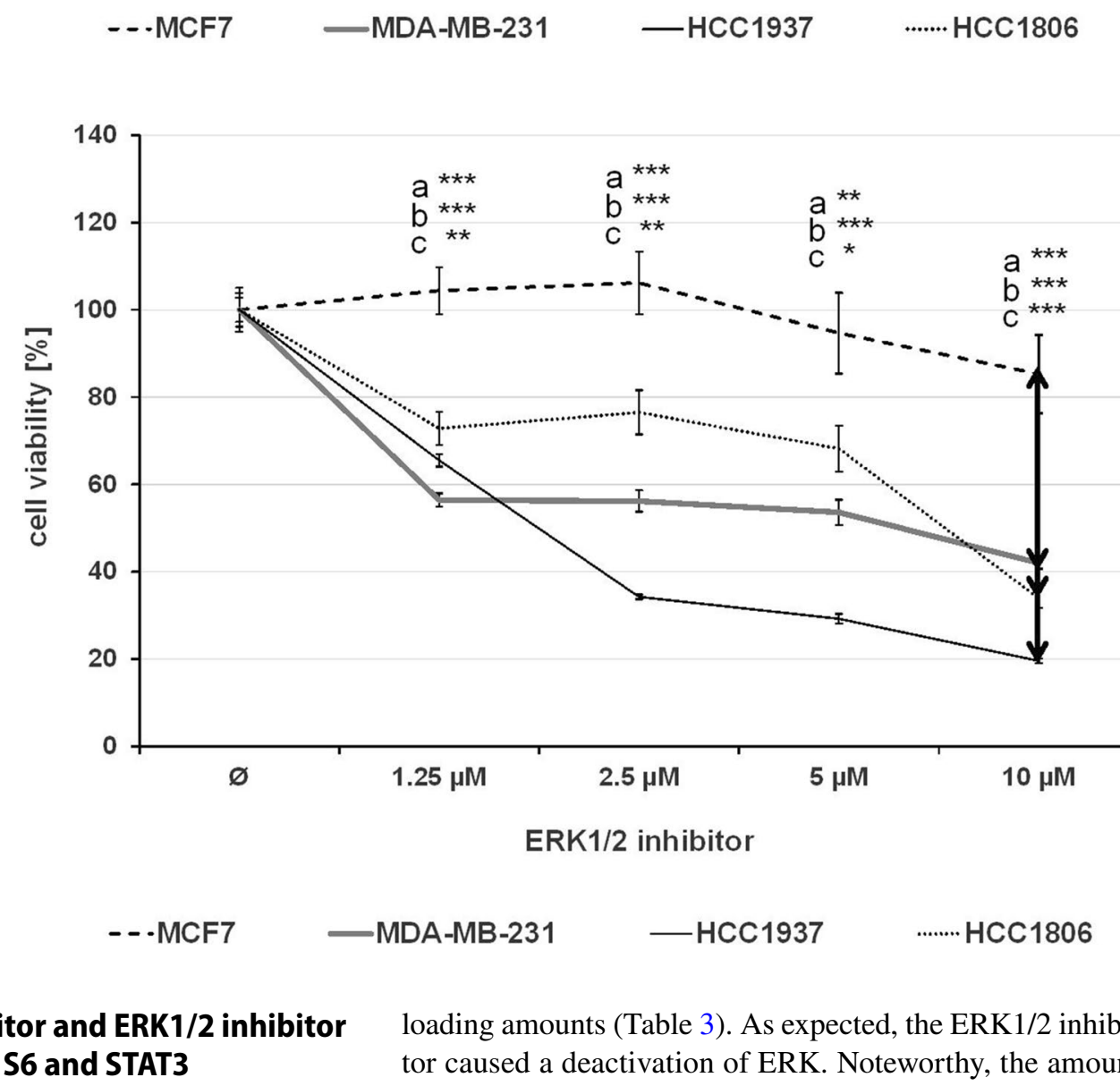

Fig. 2 MTS assay after ERK1/2 inhibitor treatment. The TNBC cells MDA-MB-231, HCC1937, HCC1806, and the non-TNBC cell line MCF7 were treated with rising concentrations of ERK1/2 inhibitor for $72 \mathrm{~h}$. Statistical significance of differences between MCF7 and a and c HCC1806: $* p<0.05$, $* * p<0.01, * * * p<0.001$ ( $t$ test $)$ MDA-MB-231, b HCC1937,

Impact of PD-1/PD-L1 inhibitor and ERK1/2
on phosphorylation of ERK, S6 and STAT3

TNBC and the non-TNBC cell lines were treated with the single inhibitors and their combination for $24 \mathrm{~h}$ and phosphorylations of ERK, S6 and STAT3 were determined by western blot analysis (Fig. 4). The percentage of phosphorylated to total protein was quantified considering protein loading amounts (Table 3). As expected, the ERK1/2 inhibitor caused a deactivation of ERK. Noteworthy, the amount of phosphorylated ERK to total ERK expression was diminished to a lower percentage in TNBC (9.1-35.5\%) than in MCF7 (63.7\%). Single treatment with the PD-1/PD-L1 inhibitor led to an increase of ERK phosphorylation in MCF7 and MDA-MB-231 cells with $154.3 \%$ and $190.2 \%$, respectively, and a reduction to $48.6 \%$ in $\mathrm{HCC} 1937$ and did 

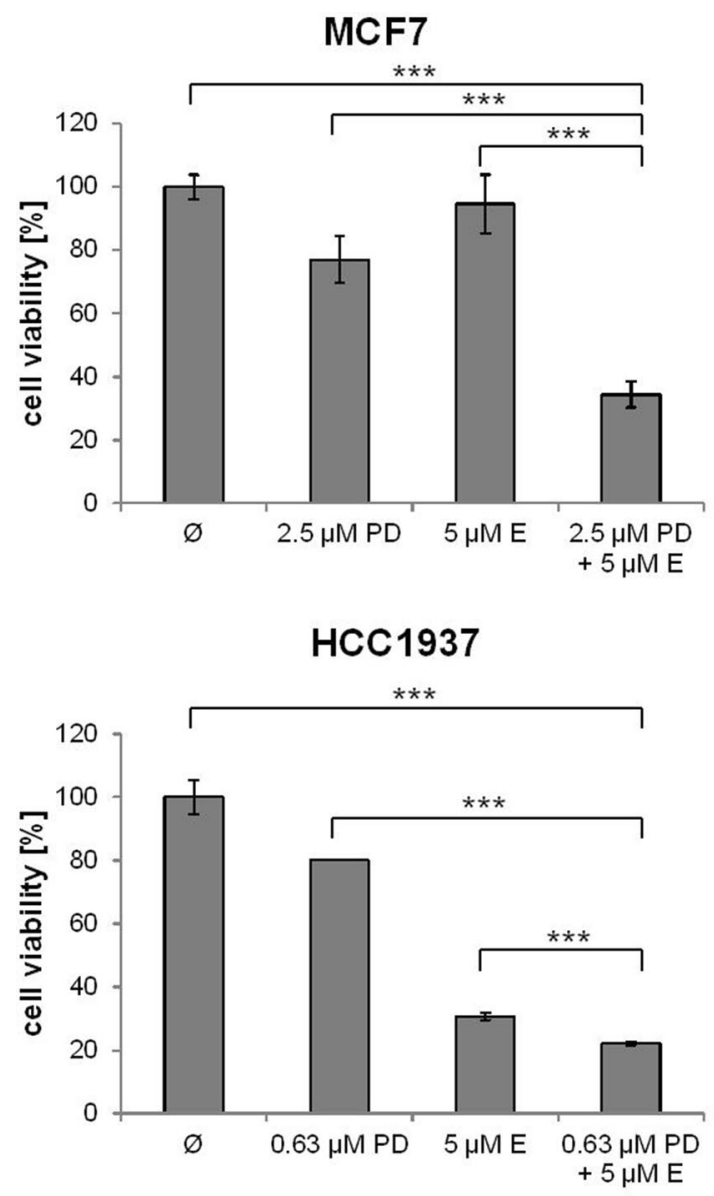

Fig. 3 MTS assay after single and combined inhibitor treatment. The MDA-MB-231, HCC1937, HCC1806, and the MCF7 cells were treated with moderate concentrations of PD-1/PD-L1 inhibitor 1 (PD)

Table 2 Calculation of interaction index values after combined treatment

\begin{tabular}{|c|c|c|c|c|}
\hline & MCF7 & MDA-MB-231 & HCC1937 & HCC1806 \\
\hline $\mathbf{y}$ & $\mathbf{0 . 7 6}$ & $\mathbf{1 . 0 2}$ & $\mathbf{0 . 8 8}$ & $\mathbf{0 . 8 5}$ \\
\hline a & 2.50 & 2.50 & 0.63 & 0.63 \\
\hline A & 3.98 & 3.37 & 2.04 & 1.73 \\
\hline b & 5.00 & 5.00 & 5.00 & 2.50 \\
\hline B & 37.13 & 17.99 & 8.69 & 5.10 \\
\hline
\end{tabular}

The TNBC cells MDA-MB-231, HCC1937, HCC1806 (white), and the non-TNBC cell line MCF7 (gray) were treated simultaneously with moderate concentrations of the PD-1/PD-L1 inhibitor $1(0.625$ or $2.5 \mu \mathrm{M})$ and the ERK1/2 inhibitor $(2.5$ or $5.0 \mu \mathrm{M})$. The interaction indexes $y(y=a / A+b / B)$ were calculated. Synergism: $y<1$; additive effect: $\mathrm{y}=1$; antagonism: $\mathrm{y}>1$

not regulate HCC1806. The combination of both inhibitors provoked a slight increase of ERK phosphorylation (116.8\%) in MCF7 cells, but a distinct dephosphorylation in all TNBC cell lines (19.9\% in MDA-MB-231, 65.2\% in HCC1937 and $57.3 \%$ in $\mathrm{HCC} 1806)$.
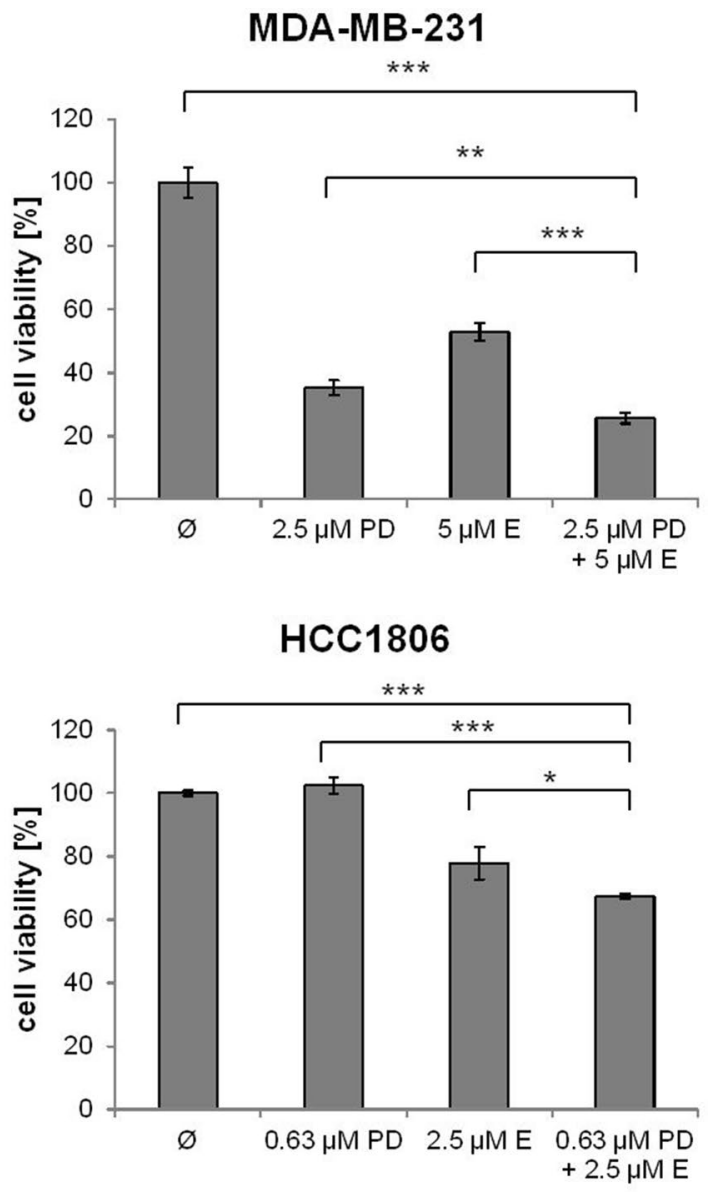

and ERK1/2 inhibitor (E) apart and in combination (PD+E) for $72 \mathrm{~h}$. Statistical significances between single and combined treatments: $* p<0.05, * * p<0.01, * * * p<0.001$ ( $t$ test)

The phosphorylation of S6 protein was enhanced after PD-1/PD-L1 inhibitor treatment in MCF7 cells, slightly decreased in MDA-MB-231 and HCC1806, and strongly reduced in HCC1937. In MCF7 the ERK1/2 inhibitor and the combined treatment significantly depleted S6 phosphorylation compared to untreated cells to 49.5 and $66.3 \%$, respectively. In all TNBC cell lines, the ERK1/2 inhibitor and the combined treatment significantly deactivated S6 with the highest content in MDA-MB-231 with 28.3 and $25.8 \%$, respectively.

The phosphorylation of STAT3 was strongly increased by PD-1/PD-L1 inhibitor treatment in MCF7 (147.3\%) and in HCC1937 (227.8\%) cells, but MDA-MB-231 and HCC1806 were nearly unchanged regarding STAT3 activation. The single ERK1/2 inhibitor and combined treatment showed no regulation of STAT3 in MCF7 and HCC1806 cells. However, in MDA-MB-231, the phosphorylation of STAT3 was increased to $282.2 \%$ and $330.6 \%$, respectively, and in $\mathrm{HCC} 1937$ to $141.6 \%$ and $135.7 \%$, respectively. 
Fig. 4 Western blots after single and combined inhibitor treatment. MCF7, MDA-MB-231, HCC1937, and HCC1806 cells were treated with $5 \mu \mathrm{M}$ PD-1/PD-L1 inhibitor 1 (PD) and $5 \mu \mathrm{M}$ ERK1/2 inhibitor (E) apart and in combination $(\mathrm{PD}+\mathrm{E})$ for $24 \mathrm{~h}$ and the phosphorylated and whole amount of ERK, S6, and STAT3 were detected in three independent experiments (one representative experiment is pictured)
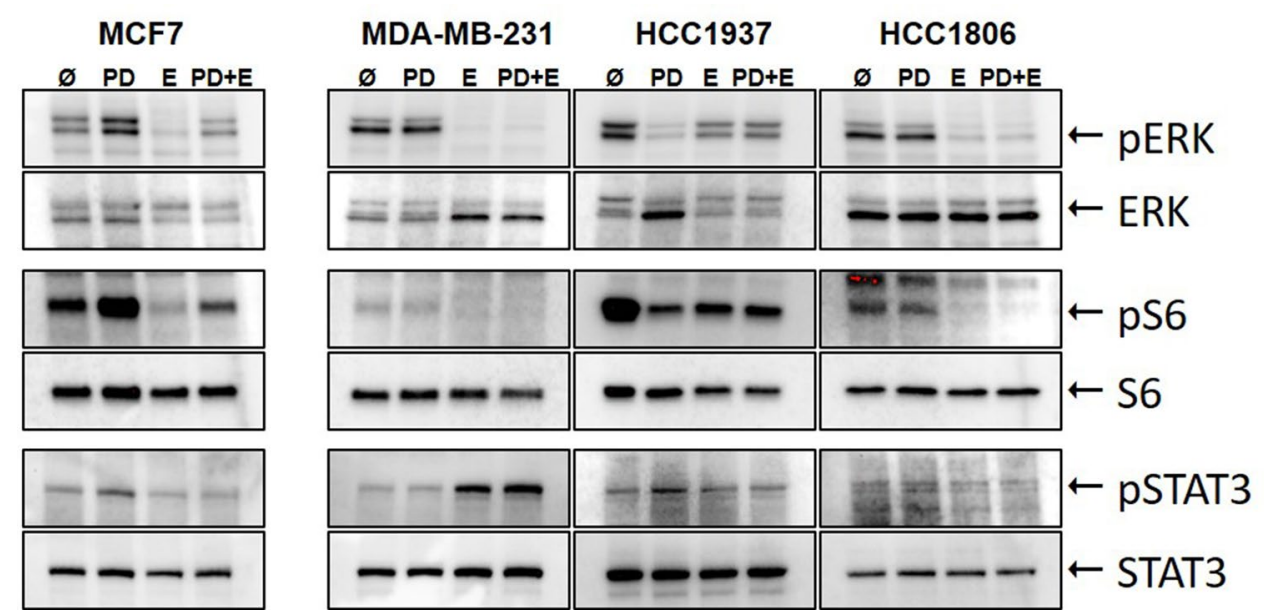

Table 3 Ratios of pERK/ERK, pS6/S6, and pSTAT3/STAT3

\begin{tabular}{|c|c|c|c|}
\hline \multicolumn{4}{|c|}{ MCF7 } \\
\hline [\%] & PD & $E$ & $P D+E$ \\
\hline pERK/ERK & $154.3 \pm 63.8$ & $63.7 \pm 29.7$ & $116.8 \pm 22.1$ \\
\hline $\mathrm{pS6/S6}$ & $156.3 \pm 41.4$ & $49.5 \pm 24.6$ * & $66.3 \pm 16.3$ * \\
\hline pSTAT3/ STAT3 & $147.3 \pm 9.9$ ** & $102.7 \pm 49.9$ & $94.1 \pm 44.6$ \\
\hline \multicolumn{4}{|c|}{ MDA-MB-231 } \\
\hline [\%] & PD & $E$ & $P D+E$ \\
\hline pERK/ERK & $190.2 \pm 95.8$ & $9.1 \pm 8.0$ *** & $19.9 \pm 15.4^{* * *}$ \\
\hline $\mathrm{pS6/S6}$ & $98.0 \pm 24.7$ & $28.2 \pm 27.1 *$ & $25.8 \pm 27.6$ ** \\
\hline pSTAT3/ STAT3 & $97.5 \pm 2.7$ & $282.2 \pm 38.8$ & $330.6 \pm 109.4$ \\
\hline \multicolumn{4}{|c|}{ HCC1937 } \\
\hline [\%] & PD & $E$ & $P D+E$ \\
\hline pERK/ERK & $48.6 \pm 78.0$ & $32.9 \pm 13.4$ *** & $65.2 \pm 50.6$ \\
\hline $\mathrm{pS6/S6}$ & $41.9 \pm 34.3^{*}$ & $44.5 \pm 11.2^{* * *}$ & $50.3 \pm 18.4^{* *}$ \\
\hline pSTAT3/ STAT3 & $227.8 \pm 143.1$ & $141.6 \pm 35.3$ & $135.7 \pm 27.5$ \\
\hline \multicolumn{4}{|c|}{ HCC1806 } \\
\hline [\%] & PD & $E$ & $P D+E$ \\
\hline pERK/ERK & $100.2 \pm 11.0$ & $35.5 \pm 9.9 * \star *$ & $57.3 \pm 31.9$ \\
\hline pS6/S6 & $91.6 \pm 5.1^{*}$ & $28.4 \pm 23.9^{* *}$ & $30.0 \pm 20.0$ ** \\
\hline pSTAT3/ STAT3 & $110.1 \pm 57.1$ & $88.8 \pm 26.9$ & $98.4 \pm 36.5$ \\
\hline
\end{tabular}

Cells were treated with either $5 \mu \mathrm{M}$ PD-1/PD-L1-Inhibitor 1 (PD), $5 \mu \mathrm{M}$ ERK1/2 inhibitor (E) or the combination of both (PD+E) for $24 \mathrm{~h}$. The percentage of phosphorylated to total protein was quantified considering protein loading amounts. Untreated cells set to $100 \%$ served as control). Results are presented as the mean \pm standard deviation of three independent experiments in percent compared to the non-treated control [\%]

Significant results in bold: ${ }^{*} \mathrm{p}<0.05, * * p<0.01, * * * p<0.001$ ( $t$ test $)$

\section{Influence of PD-1/PD-L1 inhibitor and ERK1/2 inhibitor on gene expression of immune modulatory and growth-associated genes in TNBC and the non-TNBC cell lines}

The effect of single and combined treatment of the PD-1/ PD-L1 inhibitor and the ERK1/2 inhibitor on gene expression of the immune modulatory genes of PD-L1, IL-8, and CXCR2 as well as the growth-associated genes c-Fos and FosL was investigated in all cell lines after $24 \mathrm{~h}$ of treatment by qPCR.

The relative expression rates of PD-L1, IL-8, and CXCR2 after single and combined treatment are listed in Table 4: the PD-1/PD-L1 inhibitor did not regulate the PD-L1 gene in TNBC and only non-significant to 1.76-fold in MCF7. The ERK1/2 inhibitor significantly downregulated PD-L1 in MDA-MB-231 to 0.38 and non-significantly to 0.55 in HCC1806. In HCC1937 and MCF7, PD-L1 was upregulated about twofold. The combined treatment significantly increased PD-L1 gene expression up to 20 times in the basallike cell lines HCC1937 and HCC1806, while in MDAMB-231, PD-L1 expression was three times lower and in MCF7 1.7-fold higher. The IL-8 expression was significantly downregulated to its half amount in MDA-MB-231 by the PD-1/PD-L1 inhibitor while it was upregulated in the basallike cell lines. The ERK1/2 inhibitor led to a significant upregulation of IL-8 expression both as single and combined treatment in MDA-MB-231 and HCC1806 cells. MCF7 cells showed a slight downregulation after PD-1/PD-L1 inhibitor treatment and an upregulation of IL-8 expression after ERK1/2 inhibitor and combined treatment. The CXCR2 expression was significantly downregulated by the combined treatment in MCF7, HCC1806 and MDA-MB-231, but it was upregulated in $\mathrm{HCC} 1937$.

C-Fos expression was downregulated in MCF7 and the basal-like cell lines by ERK1/2 inhibitor and combined treatment, while it was upregulated in MDA-MB-231 cells on trend. The expression of FosL was downregulated by ERK1/2 inhibitor and combined treatment in all investigated cell lines. 
Table 4 Regulation of immune modulatory and growth-associated genes after treatment with PD-1/PD-L1 and ERK1/2 inhibitors

\begin{tabular}{|c|c|c|c|}
\hline \multicolumn{4}{|c|}{ MCF7 } \\
\hline$[\mathrm{R}]$ & PD & $E$ & $P D+E$ \\
\hline PD-L1 & $1.76 \pm 0.50$ & $2.07 \pm 0.56$ & $1.70 \pm 0.48$ \\
\hline IL-8 & $0.65 \pm 0.18$ & $1.57 \pm 0.34$ & $1.21 \pm 0.16$ \\
\hline CXCR2 & $0.36 \pm 0.18$ & $0.95 \pm 0.06$ & $0.23 \pm 0.10$ * \\
\hline c-Fos & $1.17 \pm 0.09$ & $0.45 \pm 0.08$ * & $0.26 \pm 0.04$ * \\
\hline FosL & $1.26 \pm 0.16$ & $0.04 \pm 0.00$ * & $0.05 \pm 0.00$ * \\
\hline \multicolumn{4}{|c|}{ MDA-MB-231 } \\
\hline$[R]$ & PD & $E$ & $P D+E$ \\
\hline PD-L1 & $0.71 \pm 0.04$ & $0.38 \pm 0.05^{*}$ & $0.30 \pm 0.06$ \\
\hline IL-8 & $0.50 \pm 0.05$ * & $3.20 \pm 0.51$ ** & $2.62 \pm 0.47$ * \\
\hline CXCR2 & $0.47 \pm 0.22$ & $0.15 \pm 0.05$ & $0.09 \pm 0.02$ * \\
\hline c-Fos & $2.01 \pm 0.16$ * & $1.56 \pm 0.08$ & $2.94 \pm 0.06$ \\
\hline FosL & $2.84 \pm 0.50$ & $0.22 \pm 0.12$ * & $0.26 \pm 0.05$ * \\
\hline \multicolumn{4}{|c|}{ HCC1937 } \\
\hline$[R]$ & PD & $E$ & $P D+E$ \\
\hline PD-L1 & $1.44 \pm 0.05$ & $2.06 \pm 0.19$ & $20.24 \pm 1.24$ * \\
\hline IL-8 & $4.24 \pm 1.09$ & $1.46 \pm 0.24$ & $2.10 \pm 0.46$ \\
\hline CXCR2 & $0.60 \pm 0.22$ & $1.59 \pm 0.05$ & $3.58 \pm 0.02$ \\
\hline c-Fos & $0.44 \pm 0.08$ & $0.15 \pm 0.03$ * & $0.12 \pm 0.01$ ** \\
\hline FosL & $9.29 \pm 3.99$ & $0.19 \pm 0.12$ & $0.39 \pm 0.32$ \\
\hline \multicolumn{4}{|c|}{ HCC1806 } \\
\hline$[R]$ & PD & $E$ & $P D+E$ \\
\hline PD-L1 & $1.16 \pm 0.02$ * & $0.55 \pm 0.02$ & $20.04 \pm 0.38$ ** \\
\hline IL-8 & $2.43 \pm 0.18$ * & $0.37 \pm 0.01$ ** & $1.35 \pm 0.04$ * \\
\hline CXCR2 & $0.72 \pm 0.21$ & $0.86 \pm 0.13$ & $0.40 \pm 1.15$ * \\
\hline c-Fos & $0.48 \pm 0.13$ & $0.23 \pm 0.11$ * & $0.22 \pm 0.22$ \\
\hline FosL & $0.50 \pm 0.33$ & $0.14 \pm 0.02$ & $0.08 \pm 0.03$ \\
\hline
\end{tabular}

Relative gene expression ratios [R] of PD-L1, IL-8, CXCR2, c-Fos and FosL in MCF7 (gray), MDA-MB-231, HCC1937, and HCC1806 (white) after treatment with PD-1/PD-L1 inhibitor 1 (PD), ERK1/2 inhibitor $(\mathrm{E})$ and combined treatment $(\mathrm{PD}+\mathrm{E})$. Results are presented as the mean \pm standard deviation of three independent experiments in relative gene expression ratios $[R]$

Significant results in bold: ${ }^{*} \mathrm{p}<0.05,{ }^{*} p<0.01$ ( $t$ test)

\section{Discussion}

The application of immune checkpoint inhibitors in TNBC is subject of current cancer research. For a better response rate, combination treatment strategies with other antitumor agents were investigated in recent studies. Patients with TNBC response to anti-PD-1/PD-L1 therapy, and inhibitors of the MAPK/ERK signaling cascade showed impeding effect on proliferation and clinical potency in diverse cancer entities. Both signaling pathways, if activated, promote tumorigenesis on different molecular levels and therefore a combined blockage may be therapeutically promising.

In the present work, an anti-proliferative effect was confirmed on breast cancer cells by inhibitors of MAPK/ ERK and PD-1/PD-L1 signaling pathways. Both inhibitors inhibited cell viability in all investigated breast cancer cell lines. Both, the PD-1/PD-L1 inhibitor as well as the ERK1/2 inhibitor, caused half-reduced cell viability $\left(\mathrm{IC}_{50}\right)$ at lower concentrations in TNBC than in non-TNBC cell lines.

The TNBC cell lines and especially the group of basallike subtypes, HCC1937 and HCC1806, seemed to react most sensitive to both inhibitors. In clinical studies, immune checkpoint inhibitors targeting PD-1/PD-L1 applied as single treatment regime reached response rates of 4.7 to $23.1 \%$ of patients of metastasized breast cancer dependent of subtype and PD-L1 expression. Within this group, TNBC patients with PD-L1-positive tumors frequently showed the highest objective response rates (Solinas et al. 2017). Adams and colleagues reported of response rates for pembrolizumab of 5.3\% in TNBC (Adams et al. 2019b) and even 21.4\% in PD-L1-positive TNBC (Adams et al. 2019a). In our work, a specific effect on immune response could not be investigated due to the missing PD-1 component in the in vitro tumor cell model. However, the PD-1/PD-L1 inhibitor seemed to perform a direct growth inhibition in TNBC cell lines. According to the work of Cui and coworkers, downregulation of PD-L1 with siRNA led to a decreased phosphorylation of ERK and STAT3 in oral squamous cell carcinoma cell lines. Furthermore, mesenchymal proteins involved in EMT were diminished in siPD-L1 treated cells (Cui et al. 2021) providing evidence that $\mathrm{PD}-\mathrm{L} 1$ participates in proliferation and metastasis. An antitumoral effect by an inhibition of the MAPK signaling pathway in TNBC was confirmed by Nagaria and colleagues: the MEK inhibitor U0126 increased the cytotoxicity of the dual EGFR/HER2 inhibitor lapatinib additively, and of the Raf inhibitor sorafenib synergistically (Nagaria et al. 2017). Lee and colleagues demonstrated that the MEK inhibitor E6201 inhibited the colony formation rate, migration and invasion, caused cell cycle arrest, and induced apoptosis in TNBC cells (Lee et al. 2019b). In mice, E6201 inhibited tumor growth and lung metastasis, and improved survival (Lee et al. 2019b).

The treatment with the combination of PD-1/PD-L1 and ERK1/2 inhibitor achieved a synergistic inhibitory effect of proliferation in the basal-like and MCF7 cells and an additive effect in MDA-MB-231. Liu and colleagues investigated in a mouse model that combined immune checkpoint and MEK blockage led to a stronger growth inhibition in breast cancer compared to single treatment (Liu et al. 2015). Loi and coworkers showed in a TNBC mouse model that blocking the Ras/MAPK signaling pathway diminished 
the number of tumor-infiltrating $\mathrm{T}$ lymphocytes (TILs) and increased MHC and PD-L1 expression (Loi et al. 2016). This augmentation of antigen presentation that could lead to stronger immune response might be hindered by a PDL1-induced T cell checkpoint blockage. So, a combination with a PD-L/PD-L1 inhibitor could sub-serve the immune response. Consistent with this, Loi and coworker reported of an increased anti-tumoral immune response achieved by combined treatment with different MEK and PD-1/PD-L1 inhibitors in TNBC (Loi et al. 2016). The COLET study resulted in a clinical benefit for patients with metastasized TNBC treated with advanced combination of cobimetinib, paclitaxel and atezolizumab (Brufsky et al. 2019). Thus, the combination of MEK and PD-1/PD-L1 inhibitors was frequently investigated and seemed to be effective, especially in TNBC.

Evidence of an effect of PD-1/PD-L1 and ERK inhibitors was proven by analyses of intracellular processes. As expected, the ERK1/2 inhibitor impeded the phosphorylation of ERK and S6 in our cell culture experiments. Giltnane and Balko reported a higher prevalence of activated MAPK signaling pathway in TNBC and basal-like breast cancer (BLBC) compared to other breast cancer subtypes (Giltnane and Balko 2014). Additionally, several working groups of Hoeflich (Hoeflich et al. 2009), Jing (Jing et al. 2012), and Mirzoeva (Mirzoeva et al. 2009) verified that MEK inhibitors particularly blocked proliferation in TNBC and BLBC. In the current work, the activation of ERK and S6 was clearly diminished by combined inhibitor treatment assuming that the proliferation inhibition occurred by impeded MAPK signaling pathways. Interestingly, in MCF7 cells, the combined treatment with both inhibitors displayed an increased phosphorylation of ERK. As a drawback, in our work, the PD-1/PD-L1 inhibitor activated ERK in MDAMB-231 and MCF7. Black and coworker showed in breast cancer cell lines that resistance against chemo-therapeutics doxorubicin and docetaxel was increased by interaction of PD-1 and PD-L1 (Black et al. 2016). They also showed an increased activation of ERK in MDA-MB-231 by recombinant PD-1 inhibitor treatment (Black et al. 2016). However, in our study, the combined treatment with both inhibitors and the single treatment with the ERK inhibitor inhibited the ERK phosphorylation in MDA-MB-231 and the other TNBC cell lines. In the basal-like cell line HCC1937, ERK also was dephosphorylated by the PD-L1 inhibitor, only. This is in concordance to the downregulation of PD-L1 with siRNA in oral squamous cell carcinoma cell lines (Cui et al. 2021).

The oncogenes c-Fos and FosL were almost downregulated in TNBC by the combined treatment with both inhibitors. As part of the proliferation regulating transcription factor AP-1 (Saeki et al. 2009), c-Fos and FosL were described as oncogenes in different solid tumors e. g. in breast cancer (Lu et al. 2012). ERK activates the transcription of c-Fos (Saeki et al. 2009). In the present work, the ERK1/2 inhibitor reduced the c-Fos expression in all cell lines except in MDA-MB-231. FosL was reduced by all treatment including the ERK1/2 inhibitor. These reduced expressions of c-Fos and FosL might inhibit AP-1 and subsequently the proliferation. The PD-1/PD-L1 inhibitor as single treatment regulated the expression of FosL and c-Fos disparately, but did not rule over the effects of the combined treatment.

After treatment with the PD-1/PD-L1 inhibitor no or only a slight activation of STAT3 was found in TNBC or MCF7, respectively. On the other hand, Pardoll and coworkers reported of an activation of STAT3 that regulates the PD-L1 expression (Pardoll et al. 2012). Additionally, we detected a non-significant activation of STAT3 in TNBC and MCF7 cells after ERK1/2 inhibitor treatment. In contrast to our results, Sengupta and colleagues reported decreased phosphorylation of STAT3 by MEK inhibitor treatment (Sengupta et al. 1998). IL-8 gene expression is also activated by the treatments used here and IL- 8 is able to initiate the phosphorylation of STAT3 (Ma et al. 2020). STAT3 activation is a critical point for the investigation of the treatment strategy of combined treatment with PD-1/PD-L1 and ERK1/2 inhibitor. Thus, it should be further investigated. Our results showed that the anti-proliferative effect by both inhibitors is concomitant with inhibition of the MAPK, but not with the STAT3 signaling pathway.

PD-L1 is discussed as predictive marker for treatment of breast cancer with immune checkpoint inhibitors. In our work, PD-L1 expression in HCC1937 and HCC1806 was significantly increased after combined treatment, but diminished in MDA-MB-231 and HCC1806 cells after single treatment with the ERK inhibitor. In concordance, Qian and colleagues showed a decreased PD-L1 mRNA expression after ERK1/2 inhibition in bladder cancer cells (Qian et al. 2008), and Liu and coworkers confirmed a downregulation of PD-L1 expression and ERK phosphorylation in NSCLC cells after PD0325901 or U0126 treatment (Liu et al. 2020). Several studies indicated an increased PD-L1 expression in TNBC compared to non-TNBC, though the results differed strongly (Barrett et al. 2015; Bedognetti et al. 2016; Gatalica et al. 2014). Furthermore, the definition of PD-L1 positivity varies. While some authors report that higher PD-L1 expression on tumor cells is associated with prognostic factors e. g. Ki-67 (Botti et al. 2017) and better disease prognosis (Barrett et al. 2018; Botti et al. 2017; Lee et al. 2019a), others showed the opposite (Choi et al. 2018; Tomioka et al. 2018; Zhu et al. 2019). The elevated PD-L1 expression after combined treatment in basal-like TNBC in the present work is difficult to assess, because we were unable to investigate the interaction with PD-1 and the tumor environment. A stronger expression 
of PD-L1 may be predictive for a better response rate to PD-1/PD-L1 therapy (Solinas et al. 2017).

Further immune modulatory genes were regulated after combined treatment of PD-L/PD-L1 and ERK1/2 inhibitor, e.g. IL-8 and its receptor CXCR2. IL-8 is involved in development and progress of TNBC (Hartman et al. 2013). The inhibition of IL-8 impairs migration and cell survival of TNBC cell lines (Fu and Lin, 2018). Therefore, the reduced expression of these genes may add to the efficiency of the tumor therapy. The downregulations of immune suppressing cytokines after inhibition of the Ras/MAPK/ERK signaling pathway were reported in different tumor cells of melanoma (Sumimoto et al. 2006), head and neck squamous cell carcinoma (Mohan et al. 2015) and breast cancer (Liu et al. 2017a, b). Here, IL-8 expression was significantly reduced in only the basal-like TNBC cell line HCC1806 after ERK1/2 inhibitor treatment. With the combination of PD-1/PD-L1 inhibition that blocks the immune modulation and the downregulation of IL-8 and its receptor CXCR2 by the ERK1/2 inhibitor, both medications could synergistically reanimate the immune system to target these TNBC subtypes. Kim and colleagues found significant higher expression of IL-8 in TNBC compared to non-TNBC which could be suppressed by the MEK inhibitor U0126 (Kim et al. 2016). Rody and coworkers observed that a lower IL-8 expression is accomplished with a better prognosis of TNBC patients (Rody et al. 2011). IL-8 promotes the secretion of growth factors by TILs (Waugh and Wilson, 2008), and thus, the proliferation of tumor cells. The expression of the CXCR2 receptor was significantly reduced by the combined treatment of PD-1/PD-L1 and ERK1/2 inhibitor in all investigated cell lines except the HCC1937. A meta-analysis of 4.012 patients with solid tumors showed a negative effect of an increased CXCR2 expression for the overall survival (Yang et al. 2017). Additionally, IL-8 is able to lead to chemotherapeutic resistance by autocrine secretion by its receptor CXCR2 (Al-Khalaf et al. 2019; Ha et al. 2017). The reduced CXCR2 expression after combined inhibitor treatment may improve prognosis of survival and counteract chemoresistance. It could be a drawback that the combination of ERK1/2 inhibitor with PD-1/PD-L1 inhibitor significantly increased IL-8 expression in MDA-MB-231 and HCC1806 in the present investigation. Possibly, the upregulation is clinically not relevant for the disease course. Overexpressed IL-8 may influence the activity of immune cells in the tumor microenvironment which has to be investigated.

Due to the different results among TNBC and non-TNBC cells as well as among TNBC subtypes, we found vague evidence, in which mechanisms caused proliferation inhibition in breast cancer cells by the PD-1/PD-L1 and ERK1/2 inhibitor. Of special interest was the question, if one inhibitor influences the signaling pathway or functional proteins of the other inhibitor, respectively, and prevent the appropriate effectiveness.

The PD-1/PD-L1 inhibitor did not essentially affect proteins of the MAPK signaling pathway. In TNBC cells, the ERK inhibitor activated STAT3 as signal protein for proliferation. Otherwise, the combination inactivated the signaling proteins ERK and S6 as well as the gene expression of c-Fos and FosL that strongly activates cell proliferation as part of the AP-1 complex. Consequently, we conclude that the combined use of PD-1/PD-L1 and ERK inhibitors may be a suitable option by the search for new therapy regimes of TNBC.

In cell culture experiments, the tumor micro-environment cannot be displayed as it is in vivo. Therefore, our investigation did not allow distinct conclusion for the clinical meaning of the combination of PD-1/PD-L1 and ERK inhibitor for breast cancer patients. Because of the importance of tumor micro-environment, e.g. the detection of cytotoxic $(\mathrm{CD} 8+)$ lymphocytes is predictive for the response to antiPD-1 therapy (Tumeh et al. 2014), the next step has to be a cell culture model, where tumor cells are combined with immune cells. This could be realized in a co-culture setting with $\mathrm{T}$ cells, tumor-associated macrophages, and natural killer cells to simulate tumor environment.

Authors' contributions $\mathrm{KB}, \mathrm{AH}$ and $\mathrm{LH}$ conceptualized the study. Material preparation, data collection and analysis were performed by $\mathrm{KB}, \mathrm{EK}, \mathrm{SP}$ and FK. The study is based on the doctoral thesis by EK. The supervision over the work was done by AR. The first draft of the manuscript was written by KB and all authors commented on it. All authors read and approved the final manuscript.

Funding Open Access funding enabled and organized by Projekt DEAL. Not applicable.

Availability of data and material Data will be available and provided by request to the corresponding author.

Code availability Not applicable.

\section{Declarations}

Conflict of interest Author A. R. hold consultant/advisory role for Roche, Pfizer, Novartis, EISAI, Pierre-Fabre. Author L. H. hold consultant/advisory role for Roche, GSK, Astra Zeneca. The other authors declare that they have no conflict of interest.

Research involving humans and/or animals Not applicable.

Open Access This article is licensed under a Creative Commons Attribution 4.0 International License, which permits use, sharing, adaptation, distribution and reproduction in any medium or format, as long as you give appropriate credit to the original author(s) and the source, provide a link to the Creative Commons licence, and indicate if changes were made. The images or other third party material in this article are included in the article's Creative Commons licence, unless indicated 
otherwise in a credit line to the material. If material is not included in the article's Creative Commons licence and your intended use is not permitted by statutory regulation or exceeds the permitted use, you will need to obtain permission directly from the copyright holder. To view a copy of this licence, visit http://creativecommons.org/licenses/by/4.0/.

\section{References}

Adams S, Loi S, Toppmeyer D et al (2019a) Pembrolizumab monotherapy for previously untreated, PD-L1-positive, metastatic triple-negative breast cancer: cohort B of the phase II KEYNOTE-086 study. Ann Oncol 30:405-411. https://doi.org/10. 1093/annonc/mdy518

Adams S, Schmid P, Rugo HS et al (2019b) Pembrolizumab monotherapy for previously treated metastatic triple-negative breast cancer: cohort A of the phase II KEYNOTE-086 study. Ann Oncol 30:397-404. https://doi.org/10.1093/annonc/mdy517

Al-Khalaf HH, Al-Harbi B, Al-Sayed A et al (2019) Interleukin-8 activates breast cancer-associated adipocytes and promotes their angiogenesis- and tumorigenesis-promoting effects. Mol Cell Biol. https://doi.org/10.1128/MCB.00332-18

Arasanz H, Gato-Canas M, Zuazo M et al (2017) PD1 signal transduction pathways in T cells. Oncotarget 8:51936-51945. https:// doi.org/10.18632/oncotarget.17232

Barrett MT, Anderson KS, Lenkiewicz E et al (2015) Genomic amplification of 9p24.1 targeting JAK2, PD-L1, and PD-L2 is enriched in high-risk triple negative breast cancer. Oncotarget 6:26483-26493. https://doi.org/10.18632/oncotarget.4494

Barrett MT, Lenkiewicz E, Malasi S et al (2018) The association of genomic lesions and PD-1/PD-L1 expression in resected triplenegative breast cancers. Breast Cancer Res 20:71. https://doi. org/10.1186/s13058-018-1004-0

Bedognetti D, Maccalli C, Bader SB et al (2016) Checkpoint inhibitors and their application in breast cancer. Breast Care (basel) 11:108-115. https://doi.org/10.1159/000445335

Black M, Barsoum IB, Truesdell P et al (2016) Activation of the PD-1/PD-L1 immune checkpoint confers tumor cell chemoresistance associated with increased metastasis. Oncotarget 7:10557-10567. https://doi.org/10.18632/oncotarget.7235

Botti G, Collina F, Scognamiglio G et al (2017) Programmed death ligand 1 (PD-L1) tumor expression is associated with a better prognosis and diabetic disease in triple negative breast cancer patients. Int J Mol Sci. https://doi.org/10.3390/ijms18020459

Brufsky A, Kim SB, Zvirbule Z et al (2019) Phase II COLET study: Atezolizumab (A) + cobimetinib (C) + paclitaxel (P)/nabpaclitaxel $(\mathrm{nP})$ as first-line (1L) treatment (tx) for patients (pts) with locally advanced or metastatic triple-negative breast cancer (mTNBC). ASCO

Cancer Genome Atlas, N (2012) Comprehensive molecular portraits of human breast tumours. Nature 490:61-70. https://doi.org/10. 1038/nature 11412

Chang Q, Bournazou E, Sansone P et al (2013) The IL-6/JAK/Stat3 feed-forward loop drives tumorigenesis and metastasis. Neoplasia 15:848-862. https://doi.org/10.1593/neo.13706

Choi SH, Chang JS, Koo JS et al (2018) Differential prognostic impact of strong PD-L1 expression and 18F-FDG uptake in triple-negative breast cancer. Am J Clin Oncol 41:1049-1057. https://doi.org/10.1097/COC.0000000000000426

Cui B, Chen J, Luo M et al (2021) PKD3 promotes metastasis and growth of oral squamous cell carcinoma through positive feedback regulation with PD-L1 and activation of
ERK-STAT1/3-EMT signalling. Int J Oral Sci 13:8. https://doi. org/10.1038/s41368-021-00112

Dent R, Trudeau M, Pritchard KI et al (2007) Triple-negative breast cancer: clinical features and patterns of recurrence. Clin Cancer Res 13:4429-4434. https://doi.org/10.1158/1078-0432. CCR-06-3045

Francisco LM, Sage PT, Sharpe AH (2010) The PD-1 pathway in tolerance and autoimmunity. Immunol Rev 236:219-242. https:// doi.org/10.1111/j.1600-065X.2010.00923.x

Fu S, Lin J (2018) Blocking interleukin-6 and interleukin-8 signaling inhibits cell viability, colony-forming activity, and cell migration in human triple-negative breast cancer and pancreatic cancer cells. Anticancer Res 38:6271-6279. https://doi.org/10. 21873/anticanres. 12983

Gatalica Z, Snyder C, Maney T et al (2014) Programmed cell death 1 (PD-1) and its ligand (PD-L1) in common cancers and their correlation with molecular cancer type. Cancer Epidemiol Biomarkers Prev 23:2965-2970. https://doi.org/10.1158/1055-9965. EPI-14-0654

Giltnane JM, Balko JM (2014) Rationale for targeting the Ras/MAPK pathway in triple-negative breast cancer. Discov Med 17:275-283

Ha H, Debnath B, Neamati N (2017) Role of the CXCL8-CXCR1/2 axis in cancer and inflammatory diseases. Theranostics 7:15431588. https://doi.org/10.7150/thno.15625

Hartman ZC, Poage GM, den Hollander P et al (2013) Growth of triple-negative breast cancer cells relies upon coordinate autocrine expression of the proinflammatory cytokines IL-6 and IL-8. Cancer Res 73:3470-3480. https://doi.org/10.1158/0008-5472. CAN-12-4524-T

Hoeflich KP, O'Brien C, Boyd Z et al (2009) In vivo antitumor activity of MEK and phosphatidylinositol 3-kinase inhibitors in basal-like breast cancer models. Clin Cancer Res 15:4649-4664. https://doi. org/10.1158/1078-0432.CCR-09-0317

Jing J, Greshock J, Holbrook JD et al (2012) Comprehensive predictive biomarker analysis for MEK inhibitor GSK1120212. Mol Cancer Ther 11:720-729. https://doi.org/10.1158/1535-7163. MCT-11-0505

Kim S, Lee J, Jeon M et al (2016) MEK-dependent IL-8 induction regulates the invasiveness of triple-negative breast cancer cells. Tumour Biol 37:4991-4999. https://doi.org/10.1007/ s13277-015-4345-7

Lee J, Kim DM, Lee A (2019a) Prognostic role and clinical association of tumor-infiltrating lymphocyte, programmed death ligand-1 expression with neutrophil-lymphocyte ratio in locally advanced triple-negative breast cancer. Cancer Res Treat 51:649-663. https://doi.org/10.4143/crt.2018.270

Lee J, Lim B, Pearson T et al (2019b) Anti-tumor and anti-metastasis efficacy of E6201, a MEK1 inhibitor, in preclinical models of triple-negative breast cancer. Breast Cancer Res Treat 175:339-351. https://doi.org/10.1007/s10549-019-05166-3

Lehmann BD, Jovanovic B, Chen X et al (2016) Refinement of triple-negative breast cancer molecular subtypes: implications for neoadjuvant chemotherapy selection. PLoS ONE 11:e157368. https://doi.org/10.1371/journal.pone.0157368

Liu B, Fu L, Zhang C et al (2015) Computational design, chemical synthesis, and biological evaluation of a novel ERK inhibitor (BL-EI001) with apoptosis-inducing mechanisms in breast cancer. Oncotarget 6:6762-6775. https://doi.org/10.18632/oncotarget. 3105

Liu Q, Zhu H, Zhang C et al (2017a) Small GTPase RBJ promotes cancer progression by mobilizing MDSCs via IL-6. Oncoimmunology 6:e1245265. https://doi.org/10.1080/2162402X.2016. 1245265

Liu X, Zhao W, Wang W et al (2017b) Puerarin suppresses LPSinduced breast cancer cell migration, invasion and adhesion by 
blockage NF-kappaB and Erk pathway. Biomed Pharmacother 92:429-436. https://doi.org/10.1016/j.biopha.2017.05.102

Liu Z, Zhao K, Wei S et al (2020) ROS1-fusion protein induces PD-L1 expression via MEK-ERK activation in non-small cell lung cancer. Oncoimmunology 9:1758003. https://doi.org/10.1080/21624 02X.2020.1758003

Loi S, Dushyanthen S, Beavis PA et al (2016) RAS/MAPK activation is associated with reduced tumor-infiltrating lymphocytes in triplenegative breast cancer: therapeutic cooperation between MEK and PD-1/PD-L1 immune checkpoint inhibitors. Clin Cancer Res 22:1499-1509. https://doi.org/10.1158/1078-0432.CCR-15-1125

Lu D, Chen S, Tan X et al (2012) Fra-1 promotes breast cancer chemosensitivity by driving cancer stem cells from dormancy. Cancer Res 72:3451-3456. https://doi.org/10.1158/0008-5472. CAN-11-2536

Ma JH, Qin L, Li X (2020) Role of STAT3 signaling pathway in breast cancer. Cell Commun Signal 18:33. https://doi.org/10.1186/ s12964-020-0527-z

Marzec M, Zhang Q, Goradia A et al (2008) Oncogenic kinase NPM/ ALK induces through STAT3 expression of immunosuppressive protein CD274 (PD-L1, B7-H1). Proc Natl Acad Sci USA 105:20852-20857. https://doi.org/10.1073/pnas.0810958105

Meyuhas O (2015) Ribosomal protein S6 phosphorylation: four decades of research. Int Rev Cell Mol Biol 320:41-73. https://doi.org/ 10.1016/bs.ircmb.2015.07.006

Migali C, Milano M, Trapani, et al (2016) Strategies to modulate the immune system in breast cancer: checkpoint inhibitors and beyond. Ther Adv Med Oncol 8:360-374. https://doi.org/10.1177/ 1758834016658423

Mirzoeva OK, Das D, Heiser LM et al (2009) Basal subtype and MAPK/ERK kinase (MEK)-phosphoinositide 3-kinase feedback signaling determine susceptibility of breast cancer cells to MEK inhibition. Cancer Res 69:565-572. https://doi.org/10.1158/00085472.CAN-08-3389

Mohan S, Vander Broek R, Shah S et al (2015) MEK inhibitor PD-0325901 overcomes resistance to PI3K/mTOR inhibitor PF-5212384 and potentiates antitumor effects in human head and neck squamous cell carcinoma. Clin Cancer Res 21:3946-3956. https://doi.org/10.1158/1078-0432.CCR-14-3377

Nagaria TS, Shi C, Leduc C et al (2017) Combined targeting of Raf and Mek synergistically inhibits tumorigenesis in triple negative breast cancer model systems. Oncotarget 8:80804-80819. https:// doi.org/10.18632/oncotarget.20534

Nanda R, Chow LQ, Dees EC et al (2016) Pembrolizumab in patients with advanced triple-negative breast cancer: phase Ib KEYNOTE-012 study. J Clin Oncol 34:2460-2467. https://doi.org/ 10.1200/JCO.2015.64.8931

Pardoll DM (2012) The blockade of immune checkpoints in cancer immunotherapy. Nat Rev Cancer 12:252-264. https://doi.org/10. 1038/nrc3239

Patel SP, Kurzrock R (2015) PD-L1 expression as a predictive biomarker in cancer immunotherapy. Mol Cancer Ther 14:847-856. https://doi.org/10.1158/1535-7163.MCT-14-0983

Pfaffl MW (2001) A new mathematical model for relative quantification in real-time RT-PCR. Nucleic Acids Res 29:e45. https://doi.org/ 10.1093/nar/29.9.e45

Qian Y, Deng J, Geng L et al (2008) TLR4 signaling induces B7H1 expression through MAPK pathways in bladder cancer cells.
Cancer Invest 26:816-821. https://doi.org/10.1080/0735790080 1941852

Rody A, Karn T, Liedtke C et al (2011) A clinically relevant gene signature in triple negative and basal-like breast cancer. Breast Cancer Res 13:R97. https://doi.org/10.1186/bcr3035

Saeki Y, Endo T, Ide K et al (2009) Ligand-specific sequential regulation of transcription factors for differentiation of MCF-7 cells. BMC Genomics 10:545. https://doi.org/10.1186/ 1471-2164-10-545

Santen RJ, Song RX, McPherson R et al (2002) The role of mitogen-activated protein (MAP) kinase in breast cancer. J Steroid Biochem Mol Biol 80:239-256. https://doi.org/10.1016/s09600760(01)00189-3

Schmid P, Rugo HS, Adams S et al (2020) Atezolizumab plus nabpaclitaxel as first-line treatment for unresectable, locally advanced or metastatic triple-negative breast cancer (IMpassion130): updated efficacy results from a randomised, double-blind, placebo-controlled, phase 3 trial. Lancet Oncol 21:44-59. https:// doi.org/10.1016/S1470-2045(19)30689-8

Sengupta TK, Talbot ES, Scherle PA et al (1998) Rapid inhibition of interleukin- 6 signaling and Stat 3 activation mediated by mitogenactivated protein kinases. Proc Natl Acad Sci USA 95:1110711112. https://doi.org/10.1073/pnas.95.19.11107

Siegel RL, Miller KD, Jemal A (2019) Cancer statistics, 2019. CA Cancer J Clin 69:7-34. https://doi.org/10.3322/caac.21551

Solinas C, Gombos A, Latifyan S et al (2017) Targeting immune checkpoints in breast cancer: an update of early results. ESMO Open 2:e000255. https://doi.org/10.1136/esmoopen-2017-000255

Sumimoto H, Imabayashi F, Iwata T et al (2006) The BRAF-MAPK signaling pathway is essential for cancer-immune evasion in human melanoma cells. J Exp Med 203:1651-1656. https://doi. org/10.1084/jem.20051848

Tomioka N, Azuma M, Ikarashi M et al (2018) The therapeutic candidate for immune checkpoint inhibitors elucidated by the status of tumor-infiltrating lymphocytes (TILs) and programmed death ligand 1 (PD-L1) expression in triple negative breast cancer (TNBC). Breast Cancer 25:34-42. https://doi.org/10.1007/ s12282-017-0781-0

Tumeh PC, Harview CL, Yearley JH et al (2014) PD-1 blockade induces responses by inhibiting adaptive immune resistance. Nature 515:568-571. https://doi.org/10.1038/nature13954

Waugh DJ, Wilson C (2008) The interleukin-8 pathway in cancer. Clin Cancer Res 14:6735-6741. https://doi.org/10.1158/1078-0432. CCR-07-4843

Yang Y, Luo B, An Y et al (2017) Systematic review and meta-analysis of the prognostic value of CXCR2 in solid tumor patients. Oncotarget 8:109740-109751. https://doi.org/10.18632/oncotarget. 22285

Zhu X, Zhang Q, Wang D et al (2019) Expression of PD-L1 attenuates the positive impacts of high-level tumor-infiltrating lymphocytes on prognosis of triple-negative breast cancer. Cancer Biol Ther 20:1105-1112. https://doi.org/10.1080/15384047.2019.1595282

Publisher's Note Springer Nature remains neutral with regard to jurisdictional claims in published maps and institutional affiliations. 